\title{
Erratum: Age-Dependency of Location of Epileptic Foci in "Continuous Spike-and-Waves during Sleep": A Parallel to the Posterior- Anterior Trajectory of Slow Wave Activity
}

Bigna Katrin Bölsterli Heinzle ${ }^{1,2}$ Thomas Bast ${ }^{3}$ Hanne Critelli ${ }^{1,2}$ Reto Huber ${ }^{2,4,5}$ Bernhard Schmitt ${ }^{1,2}$

${ }^{1}$ Division of Clinical Neurophysiology, University Children's Hospital Zurich, Zurich, Switzerland

2 Pediatric Sleep Disorders Center, University Children's Hospital Zurich, Zurich, Switzerland

3 Epilepsy Centre Kork, Kehl-Kork, Germany

${ }^{4}$ Child Development Center, University Children's Hospital Zurich, Zurich, Switzerland

${ }^{5}$ Department of Child and Adolescent Psychiatry and Psychotherapy, Psychiatric Hospital, University of Zurich, Switzerland

Neuropediatrics 2017;48:401.
Address for correspondence Dr. Bigna K. Bölsterli Heinzle, Division of Clinical Neurophysiology, University Children's Hospital Zurich, Zurich, Switzerland (e-mail: bigna.boelsterli@kispi.uzh.ch).

\section{ERRATUM}

It has been brought to our attention that the name of Bigna Katrin Bölsterli Heinzle was indexed incorrectly in the above article in the journal Neuropediatrics, Volume 48 , Number 1, 2017 (DOI: 10.1055/s-0036-1593989). The author's name was indexed as "Heinzle BK" but the correct listing should be "Bölsterli Heinzle, Bigna Katrin" or "Bölsterli Heinzle BK." 